http://jmscr.igmpublication.org/home/ ISSN (e)-2347-176x ISSN (p) 2455-0450

crossref DOI: https://dx.doi.org/10.18535/jmscr/v8i3.95

\title{
Alveolar Bone Grafting in Cleft Palate Patients - An Orthodontic Perspective
}

\author{
Authors \\ G.Sreejith Kumar, Manjith Rajan
}

Department of Orthodontics, Government Dental College, Thiruvananthapuram, Kerala, India

\begin{abstract}
Cleft lip and palate is one of the birth defects affecting new born children throughout the world with wide geographical variation of its prevalence. The treatment approach of this congenital anomaly is multidisciplinary team work which include a paediatrician, pedodontist, plastic surgeon, psychologist, speech therapist, orthodontist, oral and maxillofacial surgeon and prosthodontist. Unfortunately there is a lack of consensus exists in the management protocol available and the diversity is mainly due to the lack of higher level evidence, dictating the management by dominant team member based on their experience and diversity of the problem. One of the critical stages in cleft management is alveolar bone grafting of the cleft defect area. A thorough understanding about timing, orthodontic preparation, graft materials and post-grafting care would improve the treatment outcome in many patients. An attempt is given here to increase the efficacy of these aspects so as to achieve the best possible treatment outcome in such unfortunate children.
\end{abstract}

Keywords: Cleft palate, Secondary alveolar bone grafting, Orthodontic preparation.

\section{Introduction}

An Orthodontist's youngest patient undoubtedly would be a new born infant with a unilateral or bilateral cleft lip and palate which may be a challenge in terms of skill as well as experience of the operator. Orthodontic treatment of these cases cannot be generalised as patients presenting with wide variation in clinical presentations and problems. So a decision on timing of intervention and treatment priority has to be taken on an individual basis depends on the severity and psychosocial condition of the case. Currently cleft management is devoid of sound evidence base throughout the world and as a result there is wide diversity of surgical techniques, timing and sequencing of orthodontic treatment especially in
India where there is limited resource and documentation ${ }^{1}$. Generally orthodontic treatment carried out in cleft patients can be done in two stages. The first is orthodontic treatment as part of interdisciplinary treatment plan carried out in primary dentition and mixed dentition which would aid the surgeon to perform the different surgical procedures like alveolar bone grafting and distraction of maxilla at the proper time ${ }^{2}$. The second stage is comprehensive orthodontic treatment carried out in permanent dentition for the final occlusal correction as well as to aid in surgical correction of jaws and placing prosthesis for missing tooth ${ }^{2,3}$.

Unilateral Clefts are associated usually with a minor palatal segment on cleft side which is 
collapsed towards midline due to bony defect and constriction of surgical scar occurred by previous palate repair. In untreated bilateral cleft lip and palate patients, the premaxilla maintains its anterior projection and the posterior segments tend to approximate, resulting in a narrow dental arch (Fig.1 A, B). As a consequence, anterior and posterior crossbites and maxillary arch perimeter reduction are frequently observed, and maxillary expansion is often required ${ }^{4}$. Most unilateral and bilateral alveolar clefts are associated with deficient bone, soft tissue, and missing or abnormally formed (peg, scoliosed) teeth. The most common missing tooth is the maxillary lateral incisors, although a central incisor or canine could be absent or deformed as well ${ }^{5}$.

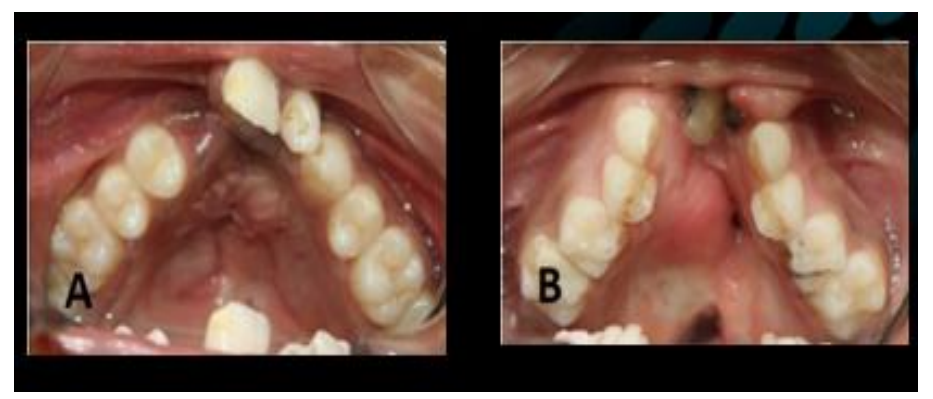

Fig.1: A-Unilateral cleft palate with constriction of affected side

B- Bilateral cleft palate with constriction of both sides

Alveolar bony defect is the main limiting factor for orthodontic treatment. Elimination of the bony defect is provided by alveolar bone grafting (ABG). Primary objective in alveolar bone grafting is to establish an intact maxilla without a break in alveolus for functional, esthetic and stability reasons. Von Eiselsberg (1901) and later Lexer (1908) were the first to use autogenous bone to graft the cleft maxilla using a pedicled osteocutaneous flap. First successful bone graft to an alveolar defect was done by Drachter in 1914 using a tibial bone graft. Milard ${ }^{6}$ in a report noted a reduced enthusiasm for grafting until 1950's. This was followed by a renewed interest in alveolar grafting in 1960's when many surgeons performed primary alveolar bone grafting in early months of life. This continued till1970's, until many reports stated that such early grafting is not advantageous as it interfere with maxillary sagittal and transverse gowth $^{7,8}$. The focus gradually shifted to a delayed bone grafting termed as secondary alveolar bone grafting (SABG) under the initiative of Oslo cleft team and the standard protocol for cleft management now introduced by Boyne and Sands 9 .

\section{Types of Alveolar grafting}

1. Primary grafting

2. Secondary grafting ${ }^{10}$
A. Early secondary $-2-5$ years
B. Early mixed dentition $-6-8$ years
C. Late mixed dentition - 9-12 years
D. Late secondary - after 13 years (after canine eruption)

E. Tertiary grafting - done in permanent dentition or along with orthognathic surgery

SABG during late mixed dentition before maxillary permanent canine eruption is the most preferred procedure now. Cho-Lee $\mathrm{G}$ et $\mathrm{al}^{11}$, has summarized the objectives of Secondary alveolar bone grafting as follows:

(1) To obtain maxillary arch continuity

(2) To maximize bone support for the dentition

(3) To stabilize the maxillary segments after orthodontic treatment, especially the mobile primary palate of bilateral clefts

(4) To eliminate oronasal fistulae

(5) To provide nasal alar cartilage support

(6) To establish ideal alveolar morphology

(7) To provide available bone with attached soft tissue for future endosteal implant placement in cases where there is a residual dental space.

\section{Timing of Bone Grafting}

Though the timing for SABG was debated, now it is almost universally accepted that the primary aim in deciding the time for SABG is to facilitate the eruption of maxillary permanent canine through the grafted bone. A chronologic age guide can be misleading at times due to its variation 
compared to dental age ${ }^{11}$. Ideal time for SABG is when half to two third of the canine root is developed as detected in a radiograph. At this stage of root development, there is accelerated and active eruption, and most of the mid face growth is complete. This corresponds to a chronologic age of 9-12 years $^{12}$. A variation of SABG timing based on presence or absence of Lateral incisor and Canine is also suggested. If lateral incisor is present and needs bone support for eruption then SABG should be done little early to preserve the health of the teeth and this usually occurs when lateral incisor is in the distal segment of the cleft. Late secondary bone grafting can be done in those who missed the SABG before canine eruption or there is failure of SABG and presence of residual oronasal fistula. This late grafting is associated with less success rate ${ }^{13}$. An early mixed dentition alveolar bone grafting (6-8 years) is sometimes considered in patients where the erupting central or lateral incisor is devoid of future bone support. Here waiting for grafting before canine eruption may jeopardize the health of incisors. Performance of SABG before eruption of permanent canine is the latest time to provide optimal outcome ${ }^{11}$. It is important to note that timing of surgery is dependent on the patient's dental development rather than chronological age.

\section{Types of grafts}

An ideal graft material for alveolar cleft reconstruction is controversial. Grafts used so far for alveolar bone grafting are autogenic, allogenic, xenogenic and alloplastic. A thorough understanding and features of available graft material for SABG are necessary for the successful treatment outcome. Most studies consider a successful outcome assessment following grafting if more than $50 \%$ of the graft bone remains 1 year after surgery. Intramembraneous bone grafts have a reported success rate of $91.67 \%$ and $83.33 \%$ for endochondral bone ${ }^{14}$. Size of initial cleft and ease of revascularization are the most important factor that decides the outcome than the type of grafts.
Donor sites for alveolar bone grafting include ileum, tibia, calvarium, mandibular symphysis, and ribs. These sites can be classified based on the bone type as intra membraneous and endochondral and based on the harvested bone as cancellous, cortical and cortico-cancellous bone. Cancellous bone of iliac crest is the gold standard autogenous graft material in $\mathrm{SABG}^{15}$. Infact all other types of grafts are compared against iliac crest graft. Animal studies have shown that endochondral bone rapidly get revascularised than intramembraneous bone, hence initial success evaluated after 6 months is greater with endochondral bone and after 6 months intramembraneous bone is well maintained than endochondralbone ${ }^{14}$. This is due to the difference in microarchitecture of the mineralized bone. Cancellous bone is considered superior to corticocancellousbone due to the ease in harvesting, presence of abundant number of osteogenic precursor cells and ease of tooth eruption through it. Cortical bone takes longer time for revascularization because it relies on vascular ingrowth by a process called "creeping substitution", whereas in cancellous bone this process is easier due to osteoconduction and osteoinduction by actual bone cells ${ }^{16}$.

\section{Orthodontic Preparation of Alveolar Bone Grafting \\ Objectives}

1. Expansion of the collapsed maxillary arch.

2. To align and derotate the anteriors which aid the surgeon for providing enough access to bone grafting at the areas of defect.

3. Superior and distal movement of premaxillary segment which is extruded in most of the bilateral cleft cases.

4. To stabilize of maxillary arch with rigid archwire or an acrylic splint which facilitate healing following grafting.

5. Extraction of supernumerary tooth on the cleft area. 
Orthodontic Expansion: Maxillary arch can be expanded sufficiently enough so that the surgeon can place a graft and be very careful in over expanding the arch as it lead to formation of oronasal fistula. Pre grafting arch expansion will improve the access to cleft for closing the nasal floor, improves post operative oral hygiene and less chance of reopening of oronasal fistula. The goal is to achieve a proper arch form by sufficient expansion. Significant segmental displacement requires pre-bone graft expansion to rotate the lateral segment(s) outward, facilitate placement of the graft, and provide the surgeon working facility during surgery. In the mixed dentition stage, arch expansion is very important because this process also normalizes the morphology and induces eruption of the canine into the symmetrical maxillary arch.

Many appliances have been used for maxillary expansion in cleft lip and palate patients, including the Quad-helix appliance, W- arch, NiTi palatal expanders, Fan type expanders and the Hyrax expander ${ }^{4}$ (Fig.2 A-D). Transverse maxillary constriction in bilateral cleft cases results in a triangular-shaped dental arch. Intercanine distance decreases more markedly during growth than does the intertuberosity distance, requiring greater expansion in the anterior region. For this reason, the expander with differential opening is recommended for achieving different anterior and posterior amounts of expansion ${ }^{17}$. Some clinicians prefer post graft arch expansion, stating that the bone consolidation is better when graft is placed under dynamic load during healing ${ }^{10}$. The other advantages are small defect to close and less volume of bone harvest needed, hence rapid bone regeneration.

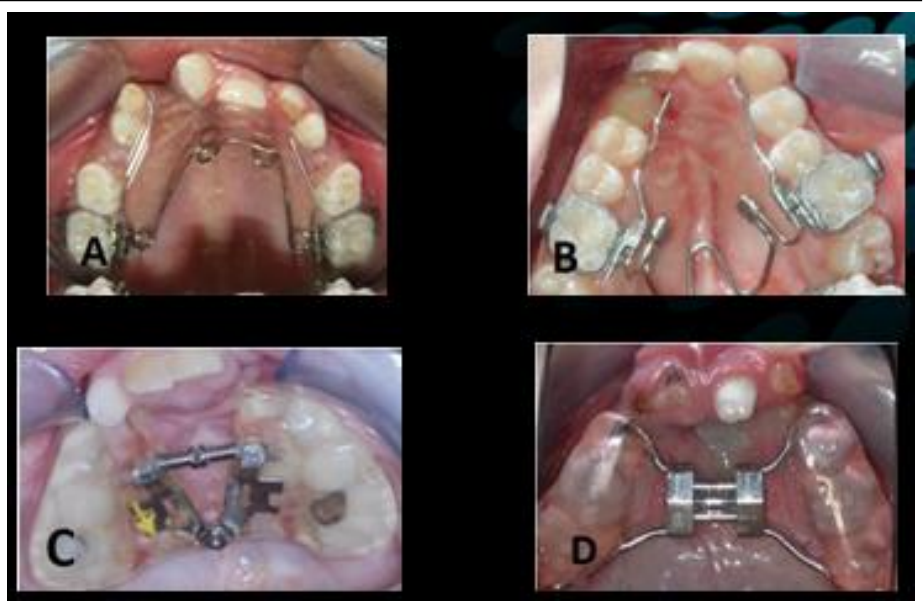

Fig.2: Orthodontic devices used for expansion in cleft palate cases. A - Quad Helix,

B - NiTi Palatal Expander, C - Fan type expander, D - Hyrax Expander

Alignment of Incisors: The tooth proximal to the cleft area may be rotated and failed to reach at the occlusal level due to lack of bone support. Derotation of incisors and correction of crossbite to the possible extent is also another perceived goal. These malpositions has to be aligned before going for alveolar bone grafting. Care should be taken in placing brackets especially for central incisors as all pre-adjusted bracket prescriptions are having a mesial crown tip and this will move the root towards the defect area which will further reduce the support and cause root resorption. It is better to keep the roots within the bone and finer root positions can be achieved after grafting ${ }^{18}$. These conditions can be eliminated by offsetting the bracket position or use of standard edgewise brackets or deferring bonding on the incisor till grafting is over.

Alignment and levelling of premaxillary segment in bilateral cleft lip and palate cases can be managed with a variety of orthodontic mechanics in association with surgical positioning in extreme cases. Since many times, the premaxillary segment is presenting with extrusion and protrusion, bypass intrusion arches are a good option. Intrusion of premaxillary segment with cantilever springs, extraoral traction, intraoral distraction devices and oral pinning and traction also can be considered. 
Stabilizing The Maxillary Arch: One of the factors in the outcome of grafting will be a more stable environment immediately after surgery which would greatly enhance the healing process by immobilising the alveolar segments with the expansion achieved. Many methods can be adopted including splinting the maxillary occlusal area with acrylic or thermoplastic material (Fig.3) at the time of surgery as well as use of passive rigid round heavy wire arches which should be inserted through the head gear tubes ${ }^{5}$.

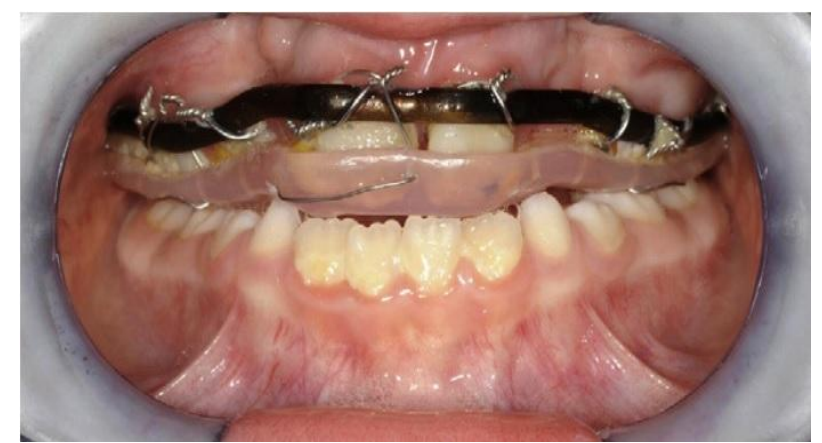

Fig.4: Acrylic splint for immobilization following SABG

Extraction of Supernumerary tooth: Many cases of cleft palate cases have supernumerary tooth in the cleft area as a routine radiographic finding. These teeth should be extracted well in advance of alveolar grafting for the successful graft intake. Generally this procedure should be done before a minimum period of six months.

\section{Conclusion}

Careful planning and team approach can produce an optimum and predictable result in SABG. A reported success rate of $90-95 \%$ in SABG proves this. A successfully grafted site is indistinguishable radiographically and endosseous implants can be placed for prosthodontic purposes at a later date. From an orthodontic perspective the need for alveolar bone grafting is very important to make the maxilla free of defect and single unit, so that orthodontic tooth movement can be carried out without compromising the periodontium.

\section{References}

1. Peter Mossey, Julian Little: Adressing the challenges of cleft lip and palate research in India: Indian J Plastic Surgery: 2009: 42: S9-S18 (1)

2. Farhad. B. Naini, Daljit. S. Gill: Orthognathic surgery: Principles,planning and practice: 2017; 799-803 (9)

3. Pradip. R. Shetye: Orthodontic management of patients with cleft lip and palate: expert Corner: 2016:6:281-286 (5)

4. Fernando Pugliesea; Juan Martin Palomob; Louise Resti Calilc; Arthur de Medeiros Alves: Dental arch size and shape after maxillary expansion in bilateral complete cleft palate: A comparison of three expander designs: Angle Orthod: 2020:90;233-238

5. Pedro E. Santiago, DMD, and Barry H. Grayson: Role of the Craniofacial Orthodontist on the Craniofacial and Cleft Lip and Palate Team: Semin Orthod; 2009; 15,225-243

6. Millard R. Cleft Craft. Vol. 3. Boston: Little, Brown; 1980: 299-301

7. Friede H, Lilja J. Dentofacial morphology in adolescent or early adult patients with cleft lip and palate after a treatment regimen that included vomer flap surgery and push back palatal repair. Scand J Plast Reconstr Surg. 1994;28:113-21.

8. Jolleys A, Robertson NR. Early bone grafting in complete clefts of lip and palate: A five years study. Br J Plast Surg 1972;25:229-37.

9. Boyne PJ, Sands NR. Secondary bone grafting of residual alveolar and palatal clefts. Journal of Oral Surgery. 1972; 30:87-92.

10. Da Silva FilhoOG, TelesSG, Ozawa TO, Filho LC. Secondary Bone Graft and Eruption of the Permanent Canine in Patients with Alveolar Clefts: Literature Review and Case Report. Angle Orthod.2000;70:174-178. 
11. Cho-Lee G, García-Díez E, Nunes R, Martí-Pagès C, Sieira-Gil R, Rivera-Baró

A. Review of secondary alveolar cleft repair. Ann Maxillo fac Surg: 2013;3:4650.

12. Bajaj AK, Wongworawat AA, Punjabi A. Management of alveolar clefts. J Craniofac Surg 2003;14 (6):840-846

13. Rune B, Jacobsson S. Dental replacement resorption after bone grafting to the alveolar cleft. Plast Reconstr Surg. 1989; 83:614-621.

14. Park Y W, Lee J H. Use of mandibular chin bone for alveolar bone grafting in cleft patients: Maxillofacial Plastic and Reconstructive Surgery 2016; 38:45.

15. Seifeldin S A. Is alveolar cleft reconstruction still controversial? The Saudi Dental Journal 2016; 28, 3-11.

16. Coots B K. Alveolar Bone Grafting: Past, Present and New Horizons; Semin Plast Surg 2012;26:178-183.

17. Garib DG, Garcia LC, Pereira V, Lauris $\mathrm{RC}$, Yen S. A rapid maxillary expander with differential opening. J Clin Orthod: 2014;48:430-435.

18. Machos, C.C. Orthodontic treatment for the cleft palate patient. Semin. Orthod. 1996; 2 (3), 197-204. 